\section{A new biliary "Y" stent for hilar tumors}

Palliative treatment of malignant hilar biliary strictures is difficult, especially when the stricture involves both the right and left hepatic ducts (Bismuth types II and higher). Drainage of both ductal systems is desirable because there is a risk of cholangitis after endoscopic biliary intervention if drainage is incomplete $[1,2]$. The rates for mean survival, 30-day mortality, and death from sepsis in patients with Klatskin tumors are reported to be significantly better with bilateral self-expanding metal stents (SEMS) compared with unilateral stents [3]. Endoscopic placement of two parallel SEMS is technically demanding. To circumvent this problem, a "Y" stent has been designed which consists of two uncovered SEMS (Niti-S Biliary stent, Y type [Endoscopic approach]; TaeWoong Medical Co. Ltd., Kyonggi-Do, South Korea): the first SEMS has a radiologically marked segment with wider mesh holes in its middle part, through which the second stent is delivered on the other side (Figure $\mathbf{1}$ ). We report a case of hilar cholangiocarcinoma (Bismuth type III), which was successfully palliatively treated using this biliary $\mathrm{Y}$ stent.

A 66-year-old woman presented with jaundice of 3 weeks' duration, and with weight loss and pruritus. Abdominal computed tomography confirmed the existence of a hilar mass, which was surgically unresectable. Endoscopic retrograde cholangiopancreatography revealed hilar cholangiocarcinoma with a Bismuth type III anatomical configuration (Figure 2). A guide wire (0.035-inch Jagwire; Microvasive Boston Scientific, Natick, Massachusetts, USA) was negotiated into the right biliary system. The first SEMS was deployed over this guide wire, with the wider mesh holes positioned below the bifurcation. A second Jagwire was then passed through the gap in the first SEMS into the left ductal system, over which the second SEMS was deployed in a " $\mathrm{Y}$ " fashion (Figure 3 ). This is the first case report describing the placement of a $\mathrm{Y}$ biliary stent in a hilar biliary stricture. The

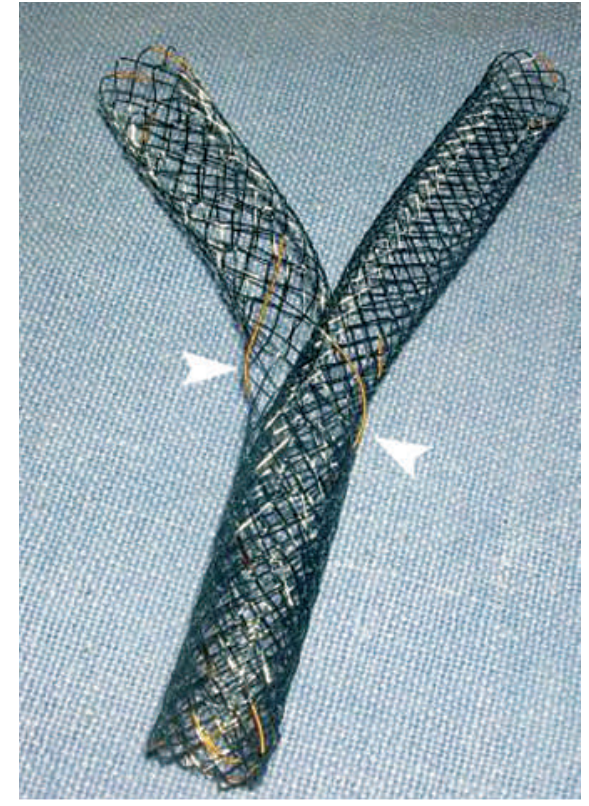

Figure 1 A biliary " $Y$ " stent with a segment of wider mesh holes in the middle part (arrowheads), through which the second stent is passed toward the contralateral side.

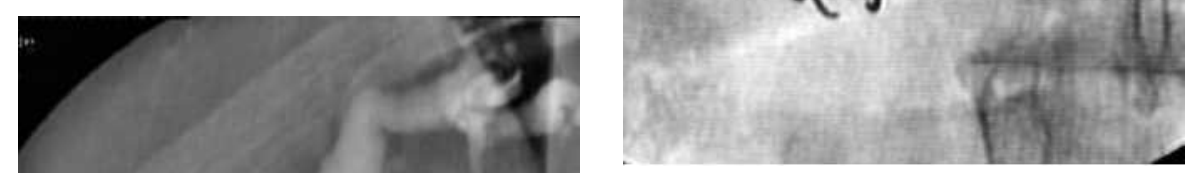

Figure 3 Radiograph showing the deployed Y stent.

patient was jaundice- and pruritis-free 10 weeks after the procedure. The Y stent appears to represent a technically easier option for the managment of malignant hilar biliary strictures.

Endoscopy_UCTN_Code_TTT_1AR_2AZ
M. Ramchandani' ${ }^{1}$, S. Lakhtakia ${ }^{1}$,
R. Gupta', M. Tandan', G. V. Rao', D. N. Reddy ${ }^{1}$ 


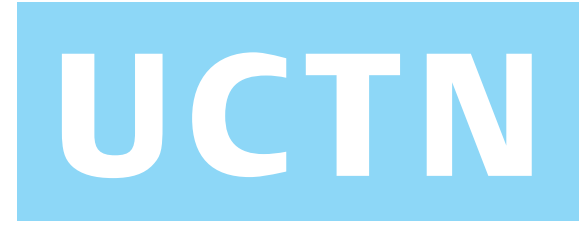

${ }^{1}$ Department of Gastroenterology, Asian Institute of Gastroenterology, Hyderabad, India

${ }^{2}$ Department of Surgical Gastroenterology, Asian Institute of Gastroenterology, Hyderabad, India.
References

${ }^{1}$ Devière J, Motte S, Dumonceau JM et al. Septicemia after endoscopic retrograde cholangiopancreatography. Endoscopy 1990; 22: $72-75$

2 Dumas R, Demuth N, Buckley M et al. Endoscopic bilateral metal stent placement for malignant hilar stenosis: identification of optimal technique. Gastrointest Endosc 2000; 51: 334-338

${ }^{3}$ Chang W, Kortan P, Haber G. Outcome in patients with bifurcation tumors who undergo unilateral versus bilateral hepatic duct drainage. Gastrointest Endosc 1998; 47: $354-362$
Corresponding author

\section{N. Reddy, MD}

Asian Institute of Gastroenterology 6-3-661 Somajiguda

Hyderabad 500082

Andhra Pradesh

India

Fax: $\quad+91-40-23324255$

Email: aigindia@yahoo.co.in 